\title{
Correction to: The CITIMERIVA Study: CITIcoline plus MEmantine plus RIVAstigmine in Older Patients Affected with Alzheimer's Disease
}

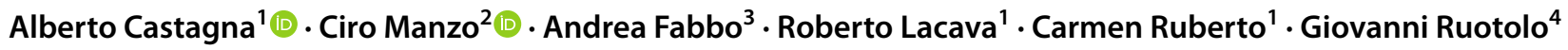

Published online: 27 February 2021

(c) Springer Nature Switzerland AG 2021

\section{Correction to:}

Clinical Drug Investigation (2021) 41:177-182

https://doi.org/10.1007/s40261-020-00996-2

The original version of this article unfortunately contained a typo in the title.

Article title, which previously read:

The CITIMERIVA Study: CITIcoline plus MEmantina plus RIVAstigmine in Older Patients Affected with Alzheimer's Disease

Should read:

The original article can be found online at https://doi.org/10.1007/ s40261-020-00996-2.

\section{Alberto Castagna}

albertocastagna@tiscali.it

1 Azienda Sanitaria Provinciale di Catanzaro, Primary Care Departiment, Center for Cognitive Disorders and Dementia, viale Crotone, 88100 Catanzaro, Italy

2 Azienda Sanitaria Locale Napoli 3 sud, Internal and Geriatric Medicine Department, Center for Cognitive Disorders and Dementia, Health District no.51, Pomigliano d'Arco, Naples, Italy

3 Azienda Unica Sanitaria Locale di Modena, Geriatric Service, Cognitive Disorders and Dementia Unit, Modena, Italy

4 Azienda Ospedaliera Pugliese-Ciaccio di Catanzaro, Geriatric Unit, Center for Cognitive Disorders and Dementia, Catanzaro, Italy

The CITIMERIVA Study: CITIcoline plus MEmantine plus RIVAstigmine in Older Patients Affected with Alzheimer's Disease 